\title{
Formation of theoretical readiness of future teachers for creative activity
}

\author{
N.N. Pashkova ${ }^{1 *}$, T.I. Oreshkina ${ }^{2}$, and I.G. Trofimova ${ }^{3}$ \\ ${ }^{1}$ I.N. Ulyanov Chuvash State University, Cheboksary, Russia \\ ${ }^{2}$ I.N. Ulyanov Chuvash State University, Cheboksary, Russia \\ ${ }^{3}$ I.N. Ulyanov Chuvash State University, Cheboksary, Russia
}

\begin{abstract}
The article discusses and describes in detail the concept of the modern content of education, which provides for training and acquisition of skills in conjunction with the formation of future specialists of the experience of creative activity. Of particular importance in the professional training of future specialists is the formation of theoretical readiness for creative learning, since the content and conditions of pedagogical activity are always probabilistic and problematic. In the process of creative learning, the teacher specifies a contradiction between the existing and the given level of learning of students, formulates a solution of this contradiction in the form of a problem. The solution of the problem is concretized in the form of a task to develop an original technology (or its elements) for solving this problem and implement it as a means of overcoming the contradiction. The necessary components of the teacher's readiness for professional creative activity are listed. All these components function as a single whole, in interaction with each other and lead to the emergence of a new system quality, which is manifested in the high quality of solving problematic pedagogical tasks in the field of education.
\end{abstract}

\section{A problem statement}

In connection with the transition to a level system of training in the higher education system, the principle of forming an active position of the student is implemented, i.e., the education of his creative thinking, intellectual abilities.

The strategy of the modern higher education system is to prepare a specialist for creative activity. The main criterion for evaluating academic performance is active activity and competence.

The basis for putting forward this strategic task for the modern education system is the recognition by the majority of specialists of the fundamental possibility of teaching creativity.

The position that the development of modern society directly depends on the creative potential of its members is generally recognized. Consequently, the creation of conditions for the development and realization of the creative potential of each individual is considered as an independent goal of the state policy in the field of education. Thanks to

${ }^{*}$ Corresponding author: prof-ped.gpa@mail.ru 
these two grounds, the course on the development of the creative personality of a specialist becomes one of the main directions of the development of higher education in modern conditions.

\subsection{The objective of the work}

Despite the adoption by universities of a socially determined target reorientation on the development of the creative personality of specialists, the work on the practical implementation of this social order is only at the very beginning.

Solving the problem of forming a creative personality requires further scientificallybased selection of the content of knowledge, improvement of the previous and development of new forms and methods of teaching, reflection in programs and textbooks of the requirements not only for the assimilation of a certain system of modernized knowledge, skills and abilities, but also for the personal qualities of students.

The theoretical and methodological foundations for solving this problem in the field of professional training of future specialists have not yet been properly reflected in the pedagogical literature [1-11].

\section{Results of the research}

Modern philosophical and psychological judgments about the meaning of creative activity are the initial basis for considering the essence of pedagogical creativity.

It is necessary to distinguish between scientific pedagogical creativity and professional pedagogical creativity [12].

The essence of pedagogical creativity is seen in the creation of new ways of influencing students. At the same time, the novelty can relate both to the content of education, and to the selection and organization of various types of educational and cognitive activities [13].

On the other hand, the creative activity of the teacher is characterized by the uniqueness of its product-the formed personality of the student in all the richness of its individual originality. Pedagogical creativity, carried out in the real educational process, is always based on the normative techniques and methods of influencing students established by previous social practice. However, their practical implementation is always mediated by the peculiarities of specific pedagogical situations, which are usually non-standard in nature [6, 9]. The variability and unconventionality of the conditions for the implementation of pedagogical activity determines the prevalence of the creative component in it.

One of the specific areas of professional activity of a teacher is [14] creative learning, which is an activity in the field of design and implementation of the didactic process, consisting in the search for new ideas and the creation of technologies (or their elements) of learning based on them. This leads to an increase in the quality and effectiveness of pedagogical activity and is a means and method of creative self-realization and selfdevelopment of the individual [15].

The legitimacy and necessity of distinguishing and considering creative learning as an independent type of pedagogical creativity, which is not reduced to creativity in the field of education or improvement of students, are due to the specifics of the subject of training, which determine the qualitative originality of didactic tasks and the features of the subject of creativity (content and learning process) that follow from these tasks [16].

Modern professional training should be aimed at forming the readiness to independently, on the basis of acquired knowledge and skills, develop and implement technologies for teaching specific actions $[3,17]$.

Readiness for creative learning determines its ability to carry out this type of pedagogical creativity [18]. 
The necessary components of the teacher's readiness for professional creative activity are:

1) personal, need-based attitude to this type of creative activity (motivational component);

2) reflexive attitude to your " I " and your activity (reflexive component);

3) knowledge of the subject bases of the performed activity (information component);

4) knowledge of how to perform subject actions and operations (operational component);

5) high level of development of creative abilities (creative component).

A high level of creativity in the field of education depends not only on the degree of development of each of these structural components [19], but also on the nature of their interaction.

Professional and creative orientation encourages and directs him to productive activities, during which there is an actualization and development of creative abilities, on the other hand, a high level of creative abilities, ensuring the quality and success of creative training, causes a sense of satisfaction and increases creative motivation [20].

Creative orientation and creative abilities are the necessary conditions for the successful functioning and further development of professional knowledge, skills and abilities.

A high level of development of motivational, creative, informational and operational components is a prerequisite for the actualization and development of reflexive processes, the emergence and strengthening of the focus of the consciousness of the subject of professional activity on the understanding of their own activities and their "I". In turn, a reflexive critical attitude to the performed activity and to oneself leads at the personal level to a rethinking of motives, value orientations, self - assessments, at the subject level-to a revision of the subject bases of the performed activity through the expansion and deepening of knowledge, at the operational level-to the need to master new ways of activity [20].

Knowledge acts as an indicative basis for professional creative activity, at the same time, in the process of solving problematic pedagogical situations, their further development takes place in the direction of expansion and deepening.

The operational component, combining the other components, itself, in turn, has a controlling influence on the features of their functioning and development. The methods of activity involved to solve this or that problem task determine the qualitative originality of the motives, creative abilities, reflexive processes and knowledge that are actualized in this case $[12,13]$.

The system of teacher readiness for professional creative activity has the property of integrity. Each of the structural components included in its content performs a function peculiar only to it, which is not reducible to the goals of the functioning of the others, but none of the components taken separately provides readiness for pedagogical creativity [1]. Only in interaction with each other, integrating into a single whole, as necessary and sufficient structural elements of a functional system, they lead to the emergence of a new system quality, manifested in the high quality of solving problematic pedagogical problems in the field of education.

Each of the structural components of readiness for creative learning represents a relatively independent, systemically organized whole, which has a specific role in the functioning of readiness, a qualitative peculiarity of the content (composition of elements), internal functioning, emergence and development [3].

The purpose of the motivational component is to show the teacher's creativity in teaching. Professional motives, which occupy a dominant position in the structure of the component, give pedagogical creativity a personal meaning [10].

The content of the motivational component consists of motivations that encourage the teacher to creative learning. 
Professional productive motives encourage and direct the activity of the teacher to achieve high learning outcomes.

Professional procedural motives encourage and direct the activity of the teacher to design and manage educational and cognitive activities due to the direct emotional appeal of the process itself.

Love for children determines the teacher's choice of specific objects of their activity: schoolchildren, and represents a relatively internal (in relation to professional) motive.

The interest in learning determines the teacher's choice of the content of training and is a relatively internal (in relation to professional) motive.

The motive of the desire for novelty encourages and directs the activity of the teacher to search for and create new things.

The motives of personal self-realization and personal self-development encourages and directs the activity of a person to the full disclosure and further development of their creative abilities.

The motives of external self-affirmation in the form of a motive for achieving success can, under certain circumstances, encourage the teacher to be creative, when improving the social status as a specialist is possible only through the search and creation of a new one.

The dominant role belongs to professional motives. They determine the features of the functioning of each of the motives and the motivational component as a whole. Due to the presence of these motives, the process and high learning outcomes become personally significant, the other motives determine the choice of the object (love for children) and the subject of training, the creative nature of the learning process (creative motives).

The emergence and development of motives that encourage and guide the creative activity of the teacher is carried out by the mechanism of shifting the "motive to the goal". At the initial stage of inclusion and development of professional activity, the teacher acts at the reproductive level, prompted and guided by the action of internal procedural (emotional attractiveness of the learning process) motives. Since the conditions of pedagogical activity are variable and changeable, the process of its implementation involves a constant correction of its content, aimed at optimizing pedagogical influences. The feeling of satisfaction from high results due to the optimization of the learning process leads to the emergence of the motive for achieving success, which, gradually increasing, begins to occupy a dominant position in relation to the procedural and internal motives [4].

The next level of development of the motivational component is associated with the actions of the teacher to resolve problem situations. The collision with the contradictions of the learning process or their discretion in ordinary pedagogical situations leads to the activation of the creative search for ways to resolve them. At first, this activity is motivated and guided by the motives of success, then the dominant role passes to the motives of creativity: motives of personal self-realization and the desire for novelty. The desire for personal self-realization and the desire to discover and create new things begin to encourage and direct the activity of the teacher to creatively solve learning problems, regardless of the desire to achieve high results [5].

Thus, in the development of the motivational component of the teacher's readiness for professional creativity, three qualitatively different levels are distinguished.

Reproductive level-the dominant motivational determinants of the learning process are professional procedural motives, love for children and interest in professional activities.

The level of optimization - in the motivational structure, the motive for achieving success in the field of professional activity arises and begins to dominate.

Creative level - in the motivational structure, the motives of personal self-realization arise and begin to dominate, and then the motives of striving for novelty in the field of professional activity. 
The purpose of the functioning of this component is to develop and update the critical attitude of the teacher to the process and results of their activities and to their "I". Thanks to the implementation of this function, human activity is directed to the dialectical negation of the ways of activity that do not correspond to the new situation, to the dialectical negation of one's own personality. Dissatisfaction with the available fund of knowledge, skills and abilities, with oneself as a person, leads to the actualization of the need to search for and discover new knowledge, new ways of activity, and personal self-development.

The content of the reflexive component consists of the reflexive processes of awareness and understanding of one's professional activity (intellectual reflection) and one's "I" in connection with the process and results of this activity (personal reflection). These types of reflection can be both retrospective and prospective (directed to the future) personality.

As a result of retrospective reflection, limited to the tasks of controlling the methods of activity used and his personality as "I", the teacher discovers their inadequacy to the content and conditions of the pedagogical situation [8].There are two possible ways of human behavior. The first is associated with the refusal to resolve the problem situation, the second - with a change in the direction of reflection on the future. On the intellectual level, this manifests itself in stops, questions and assessments, on the personal level-in doubts, selfassessments, motivations. Questions and assessments, doubts and self-assessments encourage a person to search for and discover the causes of failure, which necessarily involves the reproduction of old, unsuitable ways of activity and retrospective control in order to detect these causes.

The above connections between different types of reflection form the functional structure of the reflexive component and ensure its functioning in the system of readiness for creative activity, including learning.

The development of the reflexive component is due to the strengthening of the prospective nature of reflexive processes, first, in connection with the desire to optimize the known methods of activity, then - with the desire to solve problem situations through the discovery of new knowledge and the development of new options for action on this basis.

Taking into account this criterion, three levels of development of the reflexive component of the teacher's readiness for professional creativity are distinguished.

Reproductive level. Reflexive processes are retrospective in nature. The function of retrospective intellectual reflection is limited to self-observation of the process and the results of the learning process to ensure that they correspond to the available samples. Retrospective reflection on the personal level is manifested in the teacher's attitude to personal past experience, as sufficient to solve the pedagogical tasks facing him.

Optimization level. At this level, the teacher begins to carry out prospective reflection mainly on the intellectual level. The content and result of this type of reflection are limited to the assessment of their activities (performed according to the model) as not meeting the specific conditions of the pedagogical situation. At the personal level, reflection is still retrospective.

Creative level. Intellectual prospective reflection at this level is manifested in the active formulation of problems and the search for ways to solve them. At the personal level, there are doubts about the compliance of one's current Self with the requirements of a problematic pedagogical situation. Then, from evaluating his "I" in the context of the problem situation being solved, the FC teacher proceeds to comprehend the nature of his "personal fulfillment" as an integral "I" within a certain period of his life.

In the content of all three levels, reflexive processes of a retrospective nature are present as a mandatory element. The qualitative difference between the levels is associated with the emergence and development of prospective reflection, first at the intellectual level, and then at the personal level. 
The purpose of the functioning of the creative component of the teacher's readiness for professional creativity is to ensure the high quality of the implementation of mental functions involved in the performance of certain actions and operations included in the content of the learning process.

The content of the creative component. In accordance with the system concepts, cognitive creative activity (both on the conscious and intuitive levels) can be described by energy, spatial, temporal and informational characteristics [10]. The development and manifestation of these characteristics is determined, in accordance with the principle of unity of consciousness and activity, by the presence of a person as a subject of creative activity, adequate personal properties - creative abilities.

These abilities make up the content of the creative component of the teacher's readiness for professional creative activity.

Each of the creative abilities determines its own side of the functioning of cognitive creative activity and thus makes a specific contribution to increasing its productivity. Representing the elements of a single systemically organized whole-the ability to cognitive creative activity, they affect the development and manifestation of this integral ability not directly, but through interaction with each other.

In the development of the creative component, such inclinations as the properties of temperament (personal factor) play a significant role. The role of another factor (activity) is cognitive creative activity. The essential importance of the personal factor determines significant inter-individual differences in the level of development of creative abilities. Due to the high level of natural conditioning, creative abilities are not easily developed in the controlled educational process, and inter-individual differences in the creative component persist during the period of study at the university.

The information component. The purpose of this component is to provide the learning process with the necessary reference framework in the form of a system of knowledge and ideas.

The content of the information component consists of knowledge about the structural and functional components of the pedagogical training system, which acts as an object of management on the part of the teacher. The structural components include the purpose, content of training, subject and object of training, and the functional components include educational and cognitive activity (teaching) and teaching.

The content of training is the content of physical culture and sports activities and is presented in the form of physical exercises. In order to manage the educational and cognitive activity of students aimed at mastering this content, the teacher must know the biomechanical, physiological and psychological laws and mechanisms of development and implementation, have complete and accurate visual representations of the ways of their implementation and the motor errors committed by the students (in the process of mastering them).

In order for the learning process to be carried out on a creative level, the teacher must be equipped with knowledge about how to find and discover new knowledge through cognitive creative activity and create new, more effective pedagogical technologies (or their elements) on their basis. This type of knowledge is differentiated into three groups that differ in the degree of generalization: general methodological knowledge (dialectics of scientific knowledge), specific methodological knowledge (methodology of pedagogical research), scientific and methodological knowledge (fundamentals of scientific and methodological activities in physical culture and sports).

Knowledge about the pedagogical system of learning management and knowledge about the essence, content and methods of cognitive creative activity together constitute a necessary and sufficient number of structural elements of the information component of the teacher's readiness for creative learning. 
The development of the information component of readiness for professional pedagogical creativity is carried out in two interrelated directions: 1) content filling with new types of knowledge (expansion of the volume of knowledge); 2) development of the qualitative characteristics of the acquired knowledge in the direction of increasing their effectiveness and consistency.

For the implementation of creativity, the implementation of both directions is equally important. The development of the information component is strictly determined by the curriculum of the specialty, which determines the sequence of studying individual academic disciplines. In order to establish optimal links between individual types of knowledge, it is necessary to provide in the curriculum such a sequence of study of academic disciplines that the knowledge acquired earlier is used for the emergence, development and practical application of the knowledge studied later.

The reproductive level-the teacher can update the acquired knowledge, use it as an indicative basis for the mechanistic reproduction of known methods of activity, can theoretically justify the choice of the technology of teaching motor action or its elements based on the knowledge of sports and pedagogical disciplines.

The level of optimization - the teacher can use the knowledge as an indicative basis for reproducing the known methods of activity, taking into account the specific conditions of the pedagogical situation, can theoretically justify the choice of the technology of teaching motor action or its elements based on the knowledge of sports and pedagogical disciplines.

Creative level-the teacher can use the knowledge to develop an indicative basis for motor action, design and implement the technology (or its elements) of training, can theoretically justify their choice using general scientific and methodological knowledge.

The content of the operational component of the teacher's readiness for professional creative activity is the ability to implement individual stages of the creative learning process and the individual skills that make up their content, to perform individual didactic actions.

In the content of the operational component of readiness for creative learning, it is necessary to proceed from the ideas about the content of this type of creative activity and the didactic actions that make up their content. The quality of implementation of these actions is ensured by a high level of development of the corresponding didactic skills.

The content of creative activity is invariant and includes such functional links as 1) the stage of discretion and identification of the problem; 2) the stage of analysis of objective and subjective conditions; 3 ) the stage of hypotheses and forecasting their effectiveness; 4) the stage of goal-setting, decision-making; 5) the stage of planning; 6) the stage of practical implementation of the results of planning; 7) the stage of control and corrective actions.

The stage of identifying the problem. Creativity, including pedagogical creativity, begins with identifying the problem. The first sign of a thinking person is the ability to see problems where they are.

The following research processes are characteristic of the problem statement: 1) the detection of a contradiction, the " vision of the problem", i.e. the primary awareness of the goal with a diffuse content of conditions, which is leveled in excess information; 2) the isolation of inconsistent conditions from the point of view of the goal; 3) the actual formulation of the problem, which is a verbal and logical formalization of the contradiction, thanks to which the area of intellectual search is maximally limited.

In the creative solution of a learning task, this stage includes in its content cognitive activity aimed at detecting and highlighting in the pedagogical situation the contradiction (initial contradiction) between the initial level of readiness of students to solve certain tasks, and the level that is necessary, set from the outside. The solution of this contradiction is presented in the form of a problem, which is then concretized in the form of a pedagogical task "to teach certain actions". 
It should be emphasized that in practice, the teacher does not always face the need to identify these contradictions himself, formulate them in the form of problems and concretize them in the form of pedagogical tasks, since the educational process is carried out within the framework of the curriculum, in which the goals and objectives of training are presented initially. The solution of program problems is carried out through the use of ready-made solutions.

At the first stage of creative learning, the teacher establishes a contradiction between the existing and the given level of learning of students, formulates a solution to this contradiction in the form of a problem. The solution to the problem is specified in the form of a task to develop an original technology (or its elements) for solving this problem and implement it as a means of overcoming the contradiction.

Analysis of subjective and objective conditions. Within this stage, the teacher performs a number of qualitatively different didactic actions: studies and evaluates the mental readiness of students to assimilate this educational material [3], correlates their experience with the content of the pedagogical task, performs a psychological and pedagogical analysis of the topic being studied, taking into account its place and role in the training system.

Hypothesizing and predicting their effectiveness. The key point of this stage is to put forward various hypotheses about the ways to solve the problem, specified in the form of a creative learning task, and to predict the effectiveness of their implementation. Forecasting is based on the analysis of contradictions between the past and the present and is an attempt to look into the future, to predict the course of events, to determine and evaluate the possible effects of the implementation of the intended methods of action in the given learning conditions. To make a forecast, it is necessary to be able to present pedagogical laws and regularities in action, to know the essence of phenomena, to be able to see the internal conditionality of their changes and development, in order to take into account the possible effect of these trends in those specific conditions that may be formed or deliberately created by the teacher himself [11].

Planning is related to the teacher's actions on: 1) analysis and selection of educational material, taking into account the peculiarities of its logical structure, the nature of the content, the capabilities of students and the general goals of training; 2) selection of the type, methods and organizational forms, taking into account the content of training, the time limit, the availability of training tools, the degree of their skill and personal preferences.

The results of the professional creative activity of the teacher at this stage are: 1) a model of the content of training (in the form of a system of educational elements), taking into account educational and cognitive goals, objective and subjective conditions; 2) a model of educational and cognitive activity (in the form of a system of means, methods and organizational forms of training).

At the stage of practical implementation of the planning results, the teacher solves the problem of real transformation of the problematic pedagogical situation in the direction of the goal. At the same time, the activity of the teacher is carried out in the form of pedagogical communication. From actions in the internal intellectual plan, "in the mind", the teacher proceeds to practical actions for the management of educational and cognitive activities. According to [8], managing learning activities means monitoring the complete and correct implementation of all tasks offered to the student, monitoring the quality of learning and necessarily correcting mistakes.

At the stage of control and correction actions, the teacher performs a critical analysis of the content, the process of educational and cognitive activity of students and their own activities during the implementation of the training technology; and also makes constructive changes to the training technology developed on the basis of original ideas. 
All stages of the process of managing the solution of the problematic pedagogical task of teaching motor action are complex organizations that include the previous stages in a modified, transformed form.

The level of practical knowledge of the ways of implementing these stages and the ways of performing the didactic actions that make up their content is determined by the corresponding didactic skills.

Together, they represent the necessary and sufficient number of structural elements that make up the content of the operational component of the teacher's readiness for creative learning.

The emergence and development of skills related to the later stages of the creative learning process is based on the skills implemented in the previous stages. Control and adjustment actions and the ability to perform them can be updated only after the process of practical implementation of the design results is completed. The management of educational and cognitive activity assumes the presence of the educational task, the content of the training and the project of organizing this activity. Hypotheses can be proposed based on the analysis of subjective objective conditions, the latter is carried out taking into account the content of the selected problem.

Corrective actions that complete the cycle of solving a creative learning task are carried out on the basis of a critical analysis and evaluation of all previous actions, starting with actions of discretion and highlighting the problem.

The development of the operational component is carried out in two interrelated directions. On the one hand, its content is enriched by mastering new didactic actions.

On the other hand, each of the acquired didactic skills becomes more and more generalized, which increases the possibility of their application to solve a wider range of creative tasks in the field of education.

\section{Conclusions}

The analysis and generalization of research materials devoted to the problem of creative learning allowed us to draw the following conclusions:

1. A necessary condition for the successful application of creative learning is the knowledge of pedagogical laws that determine the content of the system of didactic principles, which includes the principles of activity, activity of the individual, problemativeness, collectivity, unity of theory and practice.

2. One of the types of creative activity of a teacher is the design and implementation of the didactic process through the search for new ideas and the creation of learning technologies or their elements on their basis, which, on the one hand, lead to an increase in the quality and effectiveness of pedagogical activity, and which, on the other, is a way of creative self-realization and self-development of his personality.

3. The system of teacher readiness for professional creative activity has the property of integrity. Each of the structural components included in its content performs a function peculiar only to it, which is not reducible to the goals of the functioning of the others, but none of the components, taken separately, provides readiness for pedagogical creativity. Only in interaction with each other, integrating into a single whole, as necessary and sufficient elements of a functional system, they lead to the emergence of a new system quality, manifested in the high quality of solving problematic pedagogical problems in the field of education.

4. The main pedagogical conditions for the use of creative learning are: the educational and research nature of the construction of the educational process; taking into account the specifics of the content of the teacher's professional activity in the formation of didactic skills; systematic diagnosis of the level of training and education, and the ratio of its results 
to the requirements for the teacher's profession; professional orientation of training; dynamism, a variety of methods, techniques and means of teaching, their focus on creating conditions that allow you to recreate the real pedagogical situation, as close as possible to the future professional activity.

\section{References}

1. B.I. Korotyaev, Teaching processiscreative, 159 (2007)

2. N.A. Kanaeva, On the problem of personal self-awareness formation, Young Scientist, 11 (34), 2, 77-80 (2011)

3. I.I. Serdyuk, Improving the quality of education, In the collection: First steps in science: modern problems and prospects of development, 13-17 (2013)

4. E.P. Ilyin, Psychology of creativity, giftedness (2009)

5. I.A. Sharshov, The system of criteria, indicators and levels of the process of professional and creative self-development of subjects in higher education, Psychological and Pedagogical Journal Gaudeamus, 1, 15, 26-36 (2010)

6. T.I. Oreshkina, G.P. Laptina, Formation of psychophysical readiness for professional activity of foreign language faculties, In the collection: Actual problems of physical culture, sports and health: ways of their implementation, 73-78 (2019)

7. A.I. Orlov, M.G. Schneider, T.I. Oreshkina, Formation of readiness for the upcoming professional activity by means of physical education, In the collection: Actual problems of physical education and sports training of students, 167-169 (2009)

8. B.N. Glinkin, L.Sh. Pestryaeva, Forms and methods of convergence of educational activity with professional activity, Problems of modern pedagogical education, 260263 (2020)

9. L.Sh. Pestryaeva, O.A. Peshkumov, A.I. Orlov, On the problem of organizing physical education in modern conditions, Physical culture and health, 152-155 (2019)

10. L.Sh. Pestryaeva, O.A. Peshkumov, Pedagogical conditions for the formation of general cultural competence in the process of studying the discipline "physical culture and sport", Problems of modern pedagogical education, 170-173 (2019)

11. L.Sh. Pestryaeva, T.A. Shcherbakova, A.I. Ivanova, The influence of the problem method of teaching on the formation of an active attitude to educational activity, Bulletin of the Chuvash State Pedagogical University named after I.Y. Yakovlev, 132138 (2015)

12. D.Sh. Sadetdinov, Z.K. Tagirova, Model of improving professional training of higher education institutions by means of information technologies, Bulletin of Kazan Technological University, 333-336 (2012)

13. D.Sh. Sadetdinov, O.V. Ezhukova, Pedagogical regularities and didactic principles and conditions for the formation of readiness for creative professional activity, Bulletin of the Orenburg State University, 172-176 (2007)

14. G.L. Drandrov, Formation of readiness for professional creative activity in higher education institutions of physical culture, All-Russian scientific and Practical conference dedicated to the 85th anniversary of the Udmurt State University, 34-37 (2016)

15. O.A. Abdullina, General pedagogical training of teachers in the system of higher pedagogical education, 141 (1990)

16. K.A. Abulkhanova-Slavskaya, Activity and personality psychology, 335 (1980) 
17. V.I. Baidenko, Identification of the composition of the competence of university graduates as a necessary stage in the design of the State Educational System of the new generation, 54 (2006)

18. V.D. Shadrikov, A new model of a specialist: innovative training and competence approach, Higher Education Today, 8, 26-31 (2004)

19. Y.G. Tatur, Competence in the structure of the quality model of specialist training, Higher Education Today, 3, 20-26 (2004)

20. A.A. Verbitsky, O.G. Larinova, Personal and competence approaches in education: problems of integration, 334 (2011) 